\title{
Distributed Downlink Beamforming With Cooperative Base Stations
}

\author{
Boon Loong Ng, Member, IEEE, Jamie S. Evans, Member, IEEE, Stephen V. Hanly, Member, IEEE, and \\ Defne Aktas, Member, IEEE
}

\begin{abstract}
In this paper, we consider multicell processing on the downlink of a cellular network to accomplish "macrodiversity" transmit beamforming. The particular downlink beamformer structure we consider allows a recasting of the downlink beamforming problem as a virtual linear mean square error (LMMSE) estimation problem. We exploit the structure of the channel and develop distributed beamforming algorithms using local message passing between neighboring base stations. For 1-D networks, we use the Kalman smoothing framework to obtain a forward-backward beamforming algorithm. We also propose a limited extent version of this algorithm that shows that the delay need not grow with the size of the network in practice. For 2-D cellular networks, we remodel the network as a factor graph and present a distributed beamforming algorithm based on the sum-product algorithm. Despite the presence of loops in the factor graph, the algorithm produces optimal results if convergence occurs.
\end{abstract}

Index Terms-Cooperative base stations, distributed algorithm, downlink beamforming, Kalman smoothing, linear mean square error (LMMSE), localized interference, message passing, multicell processing, multiple-input-multiple-output (MIMO), sum-product algorithm.

\section{INTRODUCTION}

D OWNLINK beamforming in cellular systems has been an active area of research for many years. Recently, there has been a rapidly growing interest in the area of multicell processing, in which base stations cooperate to provide networkwide, macroscopic beamforming [1]-[13]. The potential gains are indeed very promising [7]. In this context, it is of great practical interest to find distributed forms of multicell cooperation.

The simplest conceptual approach to multicell processing is to assume that there is a central processing unit, or controller, to which all the base stations are connected. The central processing unit's role is to process information from all the base stations

Manuscript received December 01, 2006; revised August 22, 2008. Current version published November 21, 2008. This work was supported by the Australian Research Council. The material in this paper was presented in part at the 2005 IEEE International Symposium on Information Theory, Adelaide, Australia, September 2005 and the 2007 IEEE International Symposium on Information Theory, Nice, France, June 2007.

B. L. Ng was with ARC Special Research Centre for Ultra-Broadband Information Networks (CUBIN), Department of Electrical and Electronic Engineering, University of Melbourne, Vic. 3010, Australia. He is now with NEC Australia, Mulgrave, Vic. 3170, Australia (e-mail: boonl@3g.nec.com.au).

J. S. Evans and S. V. Hanly are with the ARC Special Research Centre for Ultra-Broadband Information Networks (CUBIN), Department of Electrical and Electronic Engineering, University of Melbourne, Vic. 3010, Australia (e-mail: j.evans@ee.unimelb.edu.au; s.hanly@ee.unimelb.edu.au).

D. Aktas is with Department of Electrical and Electronics Engineering, Bilkent University, Bilkent, Ankara 06800, Turkey (e-mail: daktas@ee.bilkent. edu.tr).

Communicated by P. Viswanath, Associate Editor for Communications.

Color version of Figure 5 in this paper is available online at http://ieeexplore. ieee.org.

Digital Object Identifier 10.1109/TIT.2008.2006426 and to provide them with the appropriate signals to transmit. However, there are several drawbacks to such an architecture. The central processing unit is a single point of failure. As a network expands, it is expensive to require each new base station (BS) to be connected directly to the central processing unit. It is of interest then to consider architectures in which base stations communicate directly with their neighbors.

In this paper, we demonstrate ways to implement the downlink beamformer in a truly distributed manner via message passing between neighboring base stations. In this approach, there is no need for a central processing unit. The global beamforming computation is distributed among the BSs, and only local communication is required between adjacent BSs.

The main technical contribution in this paper is to recognize that a general transmit beamforming problem can be recast as a simple linear mean square error (LMMSE) estimation problem for a virtual model. The data vector in the beamforming problem becomes the observation vector in the estimation problem, and the corresponding vector of LMMSE estimates provides the vector of transmitted symbols in the original beamforming problem. Because the virtual estimation problem has exactly the same structure as the LMMSE receiver for an uplink scenario, we obtain an uplink channel that is in some sense dual to the downlink beamforming channel. Indeed, we exploit prior work on distributed algorithms for data estimation in uplink models to obtain a distributed message passing algorithm for downlink beamforming.

In beamforming with a transmit antenna array, the symbol sent by an individual antenna element usually depends on the vector of data symbols to be sent by the array. This suggests that in macroscopic beamforming, a BS (or central processor) will need to know the data symbols of all the users in the entire cellular network in order to compute the appropriate symbol to transmit. We will show that this is not the case in typical cellular networks in which mobiles are in range of at most a few base stations. We will show that in such cellular systems, the amount of computation and communication, per BS, need not grow indefinitely with the size of the network.

\section{A. Related Work}

Early papers on multiple cell processing were for the uplink [14], [15]. Both these papers considered a simple linear array model for a cellular network. Wyner [15] also proposed and analyzed a hexagonal array model, and we consider the downlink version of this model in Section V. Multicell processing is sometimes called "macrodiversity" [16]. In uplink macrodiversity, base stations cooperate to jointly decode a signal from 


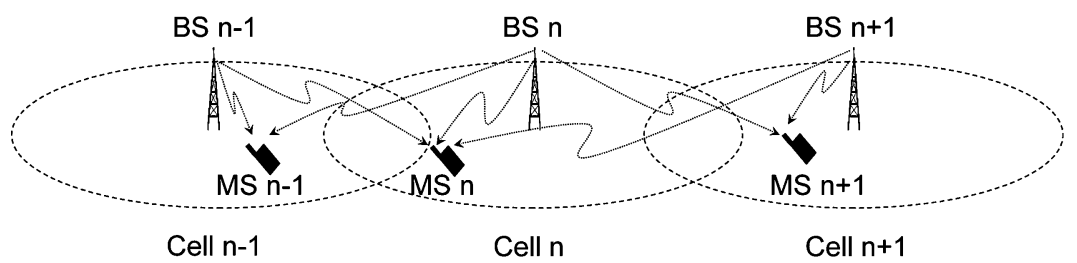

Fig. 1. Multicell downlink communication with intracell orthogonality and intercell full reuse.

a mobile in the network, taking advantage of the broadcast nature of wireless communications. In downlink macrodiversity, base stations cooperate to jointly transmit a signal to a mobile, one approach being the coherent downlink beamforming that we consider in this paper. Macrodiversity networks are a special case of multiple-input-multiple-output (MIMO), in which the multiple antennas are widely spaced across a geographic area. Macrodiversity can theoretically eliminate the impact of other cell interference on cellular capacity [7], [14], [17]. The potential capacity gain is, therefore, enormous, as demonstrated in [3], [7], and [18].

Recently, there has been renewed interest in macrodiversity for both the uplink and the downlink. Mostly, the assumption is that there is a central processor that performs the joint decoding (uplink) or joint encoding (downlink) with an infinite bandwidth backhaul network connecting the base stations to the central processor. However, recent works have begun to weaken these assumptions. In [19] and [20], global cooperation is weakened to local cooperation, in which clusters of base stations cooperate to decode the users in the cluster. In [9], [21], and [22], the impact of limited backhaul capacity is considered. The use of belief propagation and message passing between base stations is considered in [23]-[25].

\section{B. Organization of This Paper}

The multicell downlink transmission model we use in this paper is presented in Section II. In Section III, we show how the beamformer structure allows us to recast the downlink beamforming problem as a LMMSE estimation problem. Distributed transmit beamforming algorithms for the linear cellular array model are presented in Section IV, and for the hexagonal cell model in V. We conclude this paper in Section VI.

The notation used in this paper is as follows. The set of all real numbers is denoted by $\mathbb{R}$. Lower case letters are used to denote scalars, boldface lower case letters are for vectors, boldface upper case letters are for matrices, and $\boldsymbol{I}$ is the identity matrix. The $i$ th element of the vector $\boldsymbol{a}$ is $\boldsymbol{a}_{i}$ and the $\{i, j\}$ entry of the matrix $\boldsymbol{A}$ is represented by $\boldsymbol{A}_{i, j}$. Finally, we use $|\cdot|, \operatorname{tr}(\cdot),(\cdot)^{T},(\cdot)^{-1}$ and $\|\cdot\|$ to denote the absolute value, the trace, the transpose, the inverse and the Frobenius norm, respectively.

\section{Multicell Downlink Communication Model}

We consider a cellular network of $N$ cells where mobile stations (MS) within a cell do not interfere with each other, for example through time or frequency division multiplexing (i.e., TDMA or FDMA within each cell). However, we allow intercell interference by deploying full reuse in every cell. The intercell interference is assumed to be highly localized; in partic- ular, we assume that interference only comes from immediate neighbors. We focus on the multicell downlink communication process, whereby each BS wishes to transmit a data symbol to its active MS. Each BS and MS is equipped with only one antenna (this can be relaxed, to allow MIMO links, but for simplicity, we do not consider this case). For ease of expression, we refer to the $\mathrm{BS}$ and the MS in cell $n$ as BS $n$ and MS $n$, respectively. Fig. 1 illustrates a cellular network with our assumptions of local interference, intracell orthogonality, and intercell full reuse.

In addition, there are dedicated communication links, free of interference, between neighboring BSs, thus enabling a cooperative sharing of information between such BSs. Mathematically, our system model can be expressed as

$$
y_{n}=h_{n, n} x_{n}+\sum_{k \in I(n)} h_{n, k} x_{k}+w_{n}, \quad n=1, \ldots, N
$$

where $y_{n}$ is the received signal at MS $n$ and $x_{n}$ is the transmitted signal from $\operatorname{BS} n .\left\{w_{n}\right\}$ are independent identically distributed (i.i.d.) Gaussian noise variables, with zero mean and variance $\sigma^{2} . I(n)$ is the set of indices of the adjacent cells for cell $n$. The path gain from $\mathrm{BS} k$ to $\mathrm{MS} n$ is $h_{n, k}$. For simplicity, we restrict ourselves to a discrete real-valued system. The model in vector form is given by $\boldsymbol{y}=\boldsymbol{H} x+\boldsymbol{w}$, where we have defined $\boldsymbol{y} \in \mathbb{R}^{N}$ as the vector of received signals, $\boldsymbol{x} \in \mathbb{R}^{N}$ as the vector of transmitted signals, $\boldsymbol{H} \in \mathbb{R}^{N \times N}$ as the downlink channel matrix, and $\boldsymbol{w} \in \mathbb{R}^{N}$ as the noise vector.

Let $\boldsymbol{d} \in \mathbb{R}^{N}$ be the vector of $N$ scalar data symbols, where $d_{n}$ corresponds to the data symbol intended for MS $n$, which is available initially at BS $n$. In addition, $\left\{d_{n}\right\}$ are assumed to be i.i.d. random variables with zero mean and unit variance. How does the network map the data vector $\boldsymbol{d}$ into the vector of transmitted symbols $\boldsymbol{x}$ ? If we allow BS cooperation, or macrodiversity, then we can view the $N$ BSs and $N$ MSs as forming a MIMO system. Macroscopic beamforming can then be implemented via a linear precoder $\boldsymbol{T} \in \mathbb{R}^{N \times N}$ as expressed in $\boldsymbol{x}=\boldsymbol{T} \boldsymbol{d}$. Thus, the overall system is given by

$$
\boldsymbol{y}=\boldsymbol{H T d}+\boldsymbol{w} .
$$

This is the basic model of this paper, and Fig. 2 depicts this downlink cellular MIMO system.

Note that our model differs from most single-cell models with multiple colocated antennas at the BS in the structure of $\boldsymbol{H}$. In those models, $\boldsymbol{H}$ is often assumed to be a full matrix, whereas in our multicell model with localized intercell interference, $\boldsymbol{H}$ may have many zero elements. As we will see, the local coupling in the cellular network is the crucial property that allows the design of distributed transmit beamforming algorithms.

In practice, beamforming requires carrier synchronization between the separate antenna elements. This may be more diffi- 


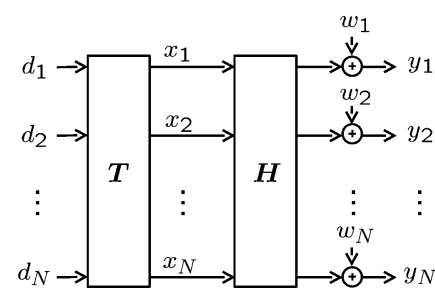

Fig. 2. MIMO model.

cult to achieve with macroscopic beamforming than with microscopic beamforming. However, if the interconnecting links between base stations are high speed with known delays, this difficulty is not insurmountable.

The following form for the precoder:

$$
\boldsymbol{T}=K \boldsymbol{H}^{T}\left(\boldsymbol{H} \boldsymbol{H}^{\boldsymbol{T}}+\beta \boldsymbol{I}\right)^{-1}
$$

with two free scalar parameters $K, \beta \in \mathbb{R}$, is a general form for many linear precoders that have been considered in the literature. By varying the choice of these parameters, it has been shown that optimality can be achieved with respect to a wide variety of criteria. For example, it arises in channel inversion and regularization [26]-[29], minimization of mean square error [30]-[34], minimization of power subject to signal-to-interference-plus noise ratio (SINR) constraints [35], and maximization of minimum SINR subject to power constraints [35]-[37]. In general, $K$ is a normalization constant used to comply with a total transmit power constraint $P_{t}$ (averaged over data symbols); if we denote $\tilde{T}$ as the unnormalized transmit beamformer, i.e.,

$$
\tilde{\boldsymbol{T}}=\boldsymbol{H}^{T}\left(\boldsymbol{H} \boldsymbol{H}^{\boldsymbol{T}}+\beta \boldsymbol{I}\right)^{-1}
$$

then $K=\sqrt{P_{t}} /\|\tilde{\boldsymbol{T}}\|$. The other parameter $\beta$ is typically a regularization parameter.

We will adopt the transmit beamforming structure (2) throughout this paper. Thus, the transmitted symbol vector will always be given by

$$
\boldsymbol{x}=K \boldsymbol{H}^{T}\left(\boldsymbol{H} \boldsymbol{H}^{\boldsymbol{T}}+\beta \boldsymbol{I}\right)^{-1} \boldsymbol{d}
$$

for an appropriate choice of $K$ and $\beta$.

\section{DOWNLINK BEAMFORMING AND A VIRTUAL LMMSE ESTIMATION PROBLEM}

In this paper, we are concerned with the problem of distributing the computation of $x$ in (4) among the transmit antenna elements. We will consider a model in which the base stations only talk to their directly connected neighbors. This avoids the need for a central processing unit, and only requires direct links between neighboring base stations. We will exploit message passing algorithms that have been developed in the area of LMMSE estimation.

The key observation of this section is that (4) resembles a well-known result in estimation theory: $\boldsymbol{x}$ can be seen as a simple scaling (by $K$ ) of the LMMSE estimate of a vector $\boldsymbol{u}$, under the model

$$
\boldsymbol{d}=\boldsymbol{H u}+\boldsymbol{z}
$$

where $\boldsymbol{u} \in \mathbb{R}^{N}$ is a vector of i.i.d. random variables with zero mean and unit variance and $z \in \mathbb{R}^{N}$ is a vector of i.i.d. random variables with zero mean and variance $\beta$, independent of $\boldsymbol{u}$. In (5), $\boldsymbol{u}$ can be interpreted as a data vector, $\boldsymbol{H}$ as a channel matrix, $\boldsymbol{d}$ as an observation data vector, and $z$ as the additive noise, in a virtual communication model.

Note that $\boldsymbol{u}$ and $\boldsymbol{z}$ have no physical meaning in terms of our original model (1). However, a consequence of the above observation is that many signal processing techniques developed for LMMSE estimation problems (e.g., [38] and [39]) are readily applicable here. We also emphasize that (5) is different from the conventional virtual uplink model used to obtain the downlink beamformer based on the duality of multiple access channel and broadcast channel [40]-[42]. This can be easily seen by noting that the channel of the conventional virtual uplink model is $\boldsymbol{H}^{T}$, not $\boldsymbol{H}$ as in our case.

\section{Distributed DOWNLINK BEAMFORMING FOR THE LINEAR CELLULAR ARRAY}

\section{A. Linear Cellular Array}

Consider first a 1-D cellular system, with channel matrix provided by

$$
\boldsymbol{H}=\left[\begin{array}{ccccc}
h_{1,1} & h_{1,2} & 0 & \ldots & 0 \\
h_{2,1} & h_{2,2} & h_{2,3} & \ldots & 0 \\
0 & h_{3,2} & h_{3,3} & \ldots & 0 \\
\vdots & & & \ddots & \\
0 & 0 & \ldots & h_{N-1, N-1} & h_{N-1, N} \\
0 & 0 & \ldots & h_{N, N-1} & h_{N, N}
\end{array}\right] .
$$

This channel model is generalized from the channel model introduced in [14] and [15] to investigate the uplink Shannon capacity of cellular networks. The band diagonal structure of the channel matrix provides a "local interaction" structure to the downlink beamforming problem, which we wish to exploit. It can be used to model BSs spaced evenly along a highway, or local network access points installed along corridors in a shopping mall, for two examples. We will extend the model to two dimensional networks in Section V.

This model has a Markov structure that allows us to propose a forward-backward algorithm for beamforming, precisely because a forward-backward estimation algorithm can be developed for the virtual uplink estimation problem. Indeed, distributed estimation algorithms have been developed for the estimation problem that arises in the original Wyner uplink model [38]. We apply these algorithms directly to the beamforming problem.

\section{B. State-Space Model and Kalman Smoothing}

We will treat $\boldsymbol{d}$ as the observation data vector on $\boldsymbol{u}$ in accordance to the channel model (5) and $\boldsymbol{x}$ as the LMMSE estimate of $\boldsymbol{u}$ given $\boldsymbol{d}$. This virtual estimation problem can be formulated as a Kalman smoothing problem by exploiting the "local interaction" structure of the cellular network.

We define the state as $v_{n}=\left[\begin{array}{lll}u_{n-1} & u_{n} & u_{n+1}\end{array}\right]^{T}$. By treating the index $n$ as "time," the evolution of $\boldsymbol{v}_{n}$ forward and backward in time can be expressed as

$$
\begin{array}{llrl}
\boldsymbol{v}_{n+1}=A^{f} \boldsymbol{v}_{n}+\boldsymbol{b}^{f} u_{n+2}, & & n=1, \ldots, N-1 \\
\boldsymbol{v}_{n-1}=A^{b} \boldsymbol{v}_{n}+\boldsymbol{b}^{b} u_{n-2}, & n=2, \ldots, N
\end{array}
$$


where

$$
\begin{aligned}
\boldsymbol{A}^{f}=\left[\begin{array}{lll}
0 & 1 & 0 \\
0 & 0 & 1 \\
0 & 0 & 0
\end{array}\right], \quad \boldsymbol{b}^{f}=\left[\begin{array}{lll}
0 & 0 & 1
\end{array}\right]^{T} \\
\boldsymbol{A}^{b}=\left[\begin{array}{lll}
0 & 0 & 0 \\
1 & 0 & 0 \\
0 & 1 & 0
\end{array}\right], \quad \boldsymbol{b}^{b}=\left[\begin{array}{lll}
1 & 0 & 0
\end{array}\right]^{T} .
\end{aligned}
$$

The observation model is given by

$$
d_{n}=\tilde{\boldsymbol{h}}_{n} \boldsymbol{v}_{n}+z_{n}, \quad n=1, \ldots, N
$$

where

$$
\begin{aligned}
\tilde{\boldsymbol{h}}_{1} & =\left[\begin{array}{llll}
0 & h_{1,1} & h_{1,2}
\end{array}\right], \quad \tilde{\boldsymbol{h}}_{N}=\left[\begin{array}{lll}
h_{N, N-1} & h_{N, N} & 0
\end{array}\right] \\
\tilde{\boldsymbol{h}}_{n} & =\left[\begin{array}{lll}
h_{n, n-1} & h_{n, n} & h_{n, n+1}
\end{array}\right], \quad i=2, \ldots, N-1 .
\end{aligned}
$$

Given the above state-space model, a forward Kalman filter can be applied [43] to produce the LMMSE estimate of the state vector $\boldsymbol{v}_{n}$, given $\left\{d_{1}, \ldots, d_{n}\right\}$ at time $n$. Similarly, the backward Kalman filter produces, at time $n$, the LMMSE estimate of the state vector $\boldsymbol{v}_{n}$, given $\left\{d_{n}, \ldots, d_{N}\right\}$. Denote $\hat{\boldsymbol{v}}_{i \mid\{j, \ldots, k\}}$ as the estimate of state $\boldsymbol{v}_{i}$ based on $\left\{d_{j}, \ldots, d_{k}\right\}$ and $\boldsymbol{M}_{i \mid\{j, \ldots, k\}}$ as its covariance. The forward and backward Kalman filters are given by the following set of equations [43].

\section{Forward Prediction}

$$
\begin{aligned}
\hat{\boldsymbol{v}}_{n \mid\{1, \ldots, n-1\}} & =A^{f} \hat{\boldsymbol{v}}_{n-1 \mid\{1, \ldots, n-1\}} \\
\boldsymbol{M}_{n \mid\{1, \ldots, n-1\}} & =A^{f} \boldsymbol{M}_{n-1 \mid\{1, \ldots, n-1\}} \boldsymbol{A}^{f^{T}}+I[n \neq N] \boldsymbol{b}^{f} \boldsymbol{b}^{f^{T}}
\end{aligned}
$$

where $I[\cdot]$ denotes the indicator function.

Forward Correction

$$
\begin{aligned}
\boldsymbol{k}_{n}^{f} & =\frac{\boldsymbol{M}_{n \mid\{1, \ldots, n-1\}} \tilde{\boldsymbol{h}}_{n}^{T}}{\beta+\tilde{\boldsymbol{h}}_{n} \boldsymbol{M}_{n \mid\{1, \ldots, n-1\}} \tilde{\boldsymbol{h}}_{n}^{T}} \\
\hat{\boldsymbol{v}}_{n \mid\{1, \ldots, n\}} & =\hat{\boldsymbol{v}}_{n \mid\{1, \ldots, n-1\}}+\boldsymbol{k}_{n}^{f}\left(d_{n}-\tilde{\boldsymbol{h}}_{n} \hat{\boldsymbol{v}}_{n \mid\{1, \ldots, n-1\}}\right) \\
\boldsymbol{M}_{n \mid\{1, \ldots, n\}} & =\left(\boldsymbol{I}-\boldsymbol{k}_{n}^{f} \tilde{\boldsymbol{h}}_{n}\right) \boldsymbol{M}_{n \mid\{1, \ldots, n-1\}} .
\end{aligned}
$$

\section{Backward Prediction}

$$
\begin{aligned}
\hat{\boldsymbol{v}}_{n \mid\{n+1, \ldots, N\}} & =\boldsymbol{A}^{b} \hat{\boldsymbol{v}}_{n+1 \mid\{n+1, \ldots, N\}} \\
\boldsymbol{M}_{n \mid\{n+1, \ldots, N\}} & =\boldsymbol{A}^{b} \boldsymbol{M}_{n+1 \mid\{n+1, \ldots, N\}} \boldsymbol{A}^{b^{T}}+\boldsymbol{b}^{b} \boldsymbol{b}^{b^{T}} I[n \neq 1]
\end{aligned}
$$

\section{Backward Correction}

$$
\begin{aligned}
\boldsymbol{k}_{n}^{b} & =\frac{\boldsymbol{M}_{n \mid\{n+1, \ldots, N\}} \tilde{\boldsymbol{h}}_{n}^{T}}{\beta+\tilde{\boldsymbol{h}}_{n} \boldsymbol{M}_{n \mid\{n+1, \ldots, N\}} \tilde{\boldsymbol{h}}_{n}^{T}} \\
\hat{\boldsymbol{v}}_{n \mid\{n, \ldots, N\}} & =\hat{\boldsymbol{v}}_{n \mid\{n+1, \ldots, N\}}+\boldsymbol{k}_{n}^{b}\left(d_{n}-\tilde{\boldsymbol{h}}_{n} \hat{\boldsymbol{v}}_{n \mid\{n+1, \ldots, N\})}\right)
\end{aligned}
$$

$$
\boldsymbol{M}_{n \mid\{n, \ldots, N\}}=\left(\boldsymbol{I}-\boldsymbol{k}_{n}^{b} \tilde{\boldsymbol{h}}_{n}\right) \boldsymbol{M}_{n \mid\{n+1, \ldots, N\}}
$$

where $\boldsymbol{k}_{n}^{f}$ and $\boldsymbol{k}_{n}^{b}$ are the Kalman gains. The forward iteration is initialized by $\hat{\boldsymbol{v}}_{1 \mid\{1\}}$ and $\boldsymbol{M}_{1 \mid\{1\}}$, while the backward iteration is initialized by $\hat{\boldsymbol{v}}_{N \mid\{N\}}$ and $\boldsymbol{M}_{N \mid\{N\}}$. These are basically "self-estimates" (estimate of $u_{n}$ based on $d_{n}$ only) and can be calculated for any $n$ as follows:

$$
\begin{aligned}
\hat{\boldsymbol{v}}_{n \mid\{n\}} & =\tilde{\boldsymbol{h}}_{n}^{T}\left(\tilde{\boldsymbol{h}}_{n} \tilde{\boldsymbol{h}}_{n}^{T}+\beta\right)^{-1} d_{n} \\
\boldsymbol{M}_{n \mid\{n\}} & =\tilde{\boldsymbol{I}}_{n}-\tilde{\boldsymbol{h}}_{n}^{T}\left(\tilde{\boldsymbol{h}}_{n} \tilde{\boldsymbol{h}}_{n}^{T}+\beta\right)^{-1} \tilde{\boldsymbol{h}}_{n}
\end{aligned}
$$

where

$$
\begin{aligned}
\tilde{\boldsymbol{I}}_{1}=\left[\begin{array}{lll}
0 & 0 & 0 \\
0 & 1 & 0 \\
0 & 0 & 1
\end{array}\right], & \tilde{\boldsymbol{I}}_{N}=\left[\begin{array}{lll}
1 & 0 & 0 \\
0 & 1 & 0 \\
0 & 0 & 0
\end{array}\right] \\
\tilde{\boldsymbol{I}}_{n}=\left[\begin{array}{lll}
1 & 0 & 0 \\
0 & 1 & 0 \\
0 & 0 & 1
\end{array}\right], & n=2, \ldots, N-1 .
\end{aligned}
$$

Using the fact that the Kalman smoother can be interpreted as linear combination of the forward and backward Kalman filters [44], the LMMSE estimate of a state $\hat{\boldsymbol{v}}_{n}$ can be written as a linear combination of $\hat{\boldsymbol{v}}_{n \mid\{n\}}, \hat{\boldsymbol{v}}_{n \mid\{1, \ldots, n-1\}}$ and $\hat{\boldsymbol{v}}_{n \mid\{n+1, \ldots, N\}}$

$$
\begin{aligned}
\hat{\boldsymbol{v}}_{n}=\boldsymbol{M}_{n} & \left(\boldsymbol{M}_{n \mid\{1, \ldots, n-1\}}^{-1} \hat{\boldsymbol{v}}_{n \mid\{1, \ldots, n-1\}}\right. \\
& \left.+\boldsymbol{M}_{n \mid\{n+1, \ldots, N\}}^{-1} \hat{\boldsymbol{v}}_{n \mid\{n+1, \ldots, N\}}+\boldsymbol{M}_{n \mid\{n\}}^{-1} \hat{\boldsymbol{v}}_{n \mid\{n\}}\right)
\end{aligned}
$$

$$
\boldsymbol{M}_{n}=\left(\boldsymbol{M}_{n \mid\{1, \ldots, n-1\}}^{-1}+\boldsymbol{M}_{n \mid\{n+1, \ldots, N\}}^{-1}+\boldsymbol{M}_{n \mid\{n\}}^{-1}-2 \tilde{\boldsymbol{I}}_{n}\right)^{-1}
$$

Based on the Kalman smoothing framework, distributed algorithms via message passing between BSs can be designed.

\section{Forward-Backward Beamforming Algorithm}

A natural distributed beamforming algorithm based on the Kalman smoothing framework is the forward-backward algorithm presented below. Assume that BS $n$ already knows $\beta$ and $\tilde{\boldsymbol{h}}_{n}$, the latter as a result of feedback from the MSs. For now, let us also assume that the scaling factor $K$ is set to unity. The algorithm is then as follows.

1) At both ends of the linear array, the initial estimates $\left\{\hat{\boldsymbol{v}}_{1 \mid\{1\}}, \boldsymbol{M}_{1 \mid\{1\}}\right\}$ and $\left\{\hat{\boldsymbol{v}}_{N \mid\{N\}}, \boldsymbol{M}_{N \mid\{N\}}\right\}$ are produced by BS 1 (given $d_{1}$ ) and BS $N$ (given $d_{N}$ ), respectively, according to (16) and (17). This is the initialization step of the forward and backward Kalman filters.

2) The outputs of BS 1 and BS $N$ are passed as messages to their direct neighbors, namely, BS 2 and BS $N-1$, respectively. This process realizes the forward prediction equations (6) and (7) for $n=2$, and the backward prediction equations (11) and (12) for $n=N-1$.

3) BS 2 and BS $N-1$ combine the messages received from their neighbors with the information from their own data $d_{2}$ and $d_{N-1}$, respectively, to produce new messages $\left\{\hat{\boldsymbol{v}}_{2 \mid\{1,2\}}, \boldsymbol{M}_{2 \mid\{1,2\}}\right\}$ and $\left\{\hat{\boldsymbol{v}}_{N-1 \mid\{N-1, N\}}, \boldsymbol{M}_{N-1 \mid\{N-1, N\}}\right\}$ according to the forward correction equations (8)-(10) and the backward correction equations (13)-(15), respectively. 


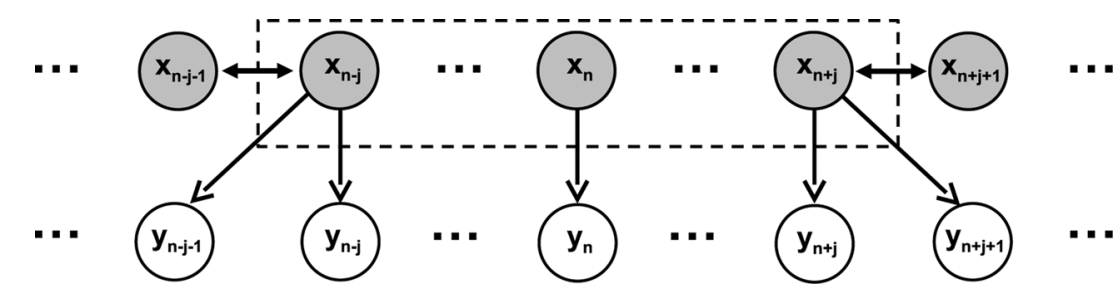

Fig. 3. Limited extent set of BSs associated with generation of $x_{n}$ after $j$ message-passing steps. Only $\left\{d_{n-j}, \cdots d_{n+j}\right\}$ are involved in producing $x_{n}$.

4) The process of information combination and message passing continues in both the forward and the backward directions until each BS has obtained its own $\hat{\boldsymbol{v}}_{n \mid\{1, \ldots, n-1\}}$, $\hat{\boldsymbol{v}}_{n \mid\{n+1, \ldots, N\}}, \hat{\boldsymbol{v}}_{n \mid\{n\}}, \boldsymbol{M}_{n \mid\{1, \ldots, n-1\}}, \boldsymbol{M}_{n \mid\{n+1, \ldots, N\}}$, and $M_{n \mid\{n\}}$.

5) For each $n$, the messages at $\mathrm{BS} n$ are then linearly combined with the local information to produce $\hat{\boldsymbol{v}}_{n}$, according to (18) and (19), and $x_{n}$ is set to be the middle component of $\hat{\boldsymbol{v}}_{n}$.

An important remark is that the distributed algorithm outlined above is sensitive to the size of the network, in particular, the time required to propagate information to cover the entire network increases linearly with the size of the cellular array. Furthermore, the delay experienced by each BS is also dependent on the location of the BS in the array. Specifically, the BSs at the edge of the array suffer longer delay than the BSs in the middle of the array. We address this issue in the following section by considering a different implementation of the Kalman message-passing algorithm that enables it to be stopped before the optimal performance is reached, allowing the delay to be independent of the size of the array.

\section{Limited Extent Distributed Beamforming Algorithm}

We exploit the local coupling property of our channel further in this section by noting that for the computation of $x_{n}$ at a particular BS $n$, information from BS $m$ decreases in importance as the distance between BS $m$ and BS $n$ increases. For this reason, there will be little impact if we replace the forward-backward algorithm with the limited extent algorithm below, which approximates the optimal solution increasingly well as the iterations increase. The algorithm is described below from the point of view of BS $n$, assuming $\beta$ and $\tilde{\boldsymbol{h}}_{n}$ are already known at BS $n$.

1) $\hat{\boldsymbol{v}}_{n \mid\{n\}}$ and $\boldsymbol{M}_{n \mid\{n\}}$ are estimated based on the data $d_{n}$ according to (16) and (17).

2) The self-estimates are sent to the BS $n-1$ and the BS $n+1$, realizing the prediction equations (6), (7), (11), and (12). At the same time, messages corresponding to the selfestimates by the neighbors $\mathrm{BS} n-1$ and $\mathrm{BS} n+1$ are received.

3) The message received from the left neighbor $\mathrm{BS} n-1$ is combined with BS $n$ 's own information $d_{n}$, producing $\hat{\boldsymbol{v}}_{n \mid\{n-1, n\}}$ and $\boldsymbol{M}_{n \mid\{n-1, n\}}$ according to the forward correction equations (8)-(10). Similarly, the message received from the right neighbor is combined with $d_{n}$, producing $\hat{\boldsymbol{v}}_{n \mid\{n, n+1\}}$ and $\boldsymbol{M}_{n \mid\{n, n+1\}}$ based on the backward correction equations (13)-(15).

4) The result from the forward correction is passed to the right neighbor $\mathrm{BS} n+1$. At the same time, the result from the backward correction is passed to the left.
5) After $j$ passes, $\hat{\boldsymbol{v}}_{n \mid\{n-j, \ldots, n+j\}}$ and $\boldsymbol{M}_{n \mid\{n-j, \ldots, n+j\}}$ can be computed using (18) and (19) with $\hat{\boldsymbol{v}}_{n \mid\{n\}}, \quad \hat{\boldsymbol{v}}_{n \mid\{n-j, \ldots, n-1\}}, \quad \hat{\boldsymbol{v}}_{n \mid\{n+1, \ldots, n+j\}}, \quad \boldsymbol{M}_{n \mid\{n\}}$ $M_{n \mid\{n-j, \ldots, n-1\}}$, and $M_{n \mid\{n+1, \ldots, n+j\}}$, where we have used the identifications

$$
\begin{aligned}
\hat{\boldsymbol{v}}_{n} & \equiv \hat{\boldsymbol{v}}_{n \mid\{n-j, \ldots, n+j\}} \\
\hat{\boldsymbol{v}}_{n \mid\{1, \ldots, n-1\}} & \equiv \hat{\boldsymbol{v}}_{n \mid\{n-j, \ldots, n-1\}} \\
\hat{\boldsymbol{v}}_{n \mid\{n+1, \ldots, N\}} & \equiv \hat{\boldsymbol{v}}_{n \mid\{n+1, \ldots, n+j\}} \\
\boldsymbol{M}_{n} & \equiv \boldsymbol{M}_{n \mid\{n-j, \ldots, n+j\}} \\
\boldsymbol{M}_{n \mid\{1, \ldots, n-1\}} & \equiv \boldsymbol{M}_{n \mid\{n-j, \ldots, n-1\}} \\
\boldsymbol{M}_{n \mid\{n+1, \ldots, N\}} & \equiv \boldsymbol{M}_{n \mid\{n+1, \ldots, n+j\}} .
\end{aligned}
$$

6) After $j$ passes, $\hat{\boldsymbol{v}}_{n \mid\{n-j, \ldots, n+j\}}$ contains an approximation $x_{n}^{(j)}$ to the optimal transmit symbol $x_{n}$.

If the number of passes $j$ is large enough for the information from the BSs at the edges of the array to arrive, then $x_{n}^{(j)}=$ $x_{n}$ is optimal. However, the algorithm can be terminated after an arbitrary number of passes, and $x_{n}^{(j)}$ incorporates only the data symbols from the limited extent set corresponding to the number of passes that have taken place (Fig. 3). If the algorithm is stopped early, optimality is lost, but delay is reduced. There is a tradeoff between performance and delay. In this version of the algorithm, the amount of knowledge about the network that a $\mathrm{BS}$ requires depends on the number of passes that have taken place when the algorithm is terminated.

Up until now, we have made the simplifying assumption that $K=1$. In some of the beamforming schemes referred to in Section II, $K$ depends on the instantaneous channels across the entire linear array; it is then a scaling factor to ensure that the total transmit energy across all BSs is conserved for all channel states. In these cases, computing $K$ requires global knowledge of the channel states, but the channel states will typically be changing much more slowly than the data symbols, so this is much less onerous than requiring global knowledge of the all the data symbols. If only the long-term average power is to be constrained, then we can set $K$ to be $\sqrt{P_{t} / \mathrm{E}\|\tilde{T}\|^{2}}$, where $\tilde{T}$ is defined in (3), and global knowledge of the channel states is not required in this case.

\section{Distributed DOWNLINK BEAMFORMING FOR HEXAGONAL CELlular ARRAY}

The 1-D cellular array model has its uses, but most cellular networks have a 2-D aspect, and the hexagonal cellular array is the traditional model of a cellular network. For this reason, we now extend the distributed beamforming model to the case of a hexagonal cellular array. The key is to recognize that the Kalman distributed algorithm is in fact a special case of the 
sum-product algorithm [45] operating on a 1-D factor graph. Interested readers should refer to [45] where the Kalman filter is proven to be a special case of the sum-product algorithm (the fact that the proof can be extended to Kalman smoothing is straightforward). In this section, we present a distributed downlink beamforming algorithm based on the sum-product algorithm that can be applied to 2-D networks with local coupling. We will use the hexagonal cellular array model [15] as an example.

\section{A. Hexagonal Cellular Array and Factor Graph}

The channel matrix $\boldsymbol{H}$ for a hexagonal cellular array can no longer be expressed as a tridiagonal matrix as in the linear cellular array. In a network of seven cells where cell 1 is surrounded by six cells labeled 2 to $7, \boldsymbol{H}$ is given by

$$
\boldsymbol{H}=\left[\begin{array}{ccccccc}
h_{1,1} & h_{1,2} & h_{1,3} & h_{1,4} & h_{1,5} & h_{1,6} & h_{1,7} \\
h_{2,1} & h_{2,2} & h_{2,3} & 0 & 0 & 0 & h_{2,7} \\
h_{3,1} & h_{3,2} & h_{3,3} & h_{3,4} & 0 & 0 & 0 \\
h_{4,1} & 0 & h_{4,3} & h_{4,4} & h_{4,5} & 0 & 0 \\
h_{5,1} & 0 & 0 & h_{5,4} & h_{5,5} & h_{5,6} & 0 \\
h_{6,1} & 0 & 0 & 0 & h_{6,5} & h_{6,6} & h_{6,7} \\
h_{7,1} & h_{7,2} & 0 & 0 & 0 & h_{7,6} & h_{7,7}
\end{array}\right] .
$$

While it is possible to construct a 2-D state-space model, we will not pursue that course here and we will instead use the model of a factor graph. A factor graph encodes a global function that can be factored into local functions, and marginalized functions can be computed using message passing on the graph. When this approach is applied to statistical inference problems, the global function is the $a$ posteriori density function, i.e., a joint probability density function (pdf) of the unknown variables conditioned on the observed ones, and the requirement is to find the marginalized probability densities given observed data.

For the virtual LMMSE estimation problem of estimating $\boldsymbol{u}$ given $\boldsymbol{d}$, we further assume that $\left\{u_{n}\right\}$ are i.i.d. Gaussian random variables with zero mean and unit variance. In this case, the LMMSE estimate $\boldsymbol{x}$ is the mean of the conditional distribution $p(\boldsymbol{u} \mid \boldsymbol{d})$, which can be factored as follows:

$$
\begin{aligned}
p(\boldsymbol{u} \mid \boldsymbol{d}) \propto & p(\boldsymbol{d} \mid \boldsymbol{u}) p(\boldsymbol{u}) \\
= & p\left(d_{1} \mid u_{1}, u_{n}, n \in I(1)\right) p\left(d_{2} \mid u_{2}, u_{n}, n \in I(2)\right) \\
& \cdots p\left(d_{N} \mid u_{N}, u_{n}, n \in I(N)\right) p\left(u_{1}\right) p\left(u_{2}\right) \cdots p\left(u_{N}\right)
\end{aligned}
$$

where $I(n)$ is the set of indices of the cells adjacent to cell $n$, e.g., for the seven cell example, we obtain

$$
\begin{aligned}
& p\left(u_{1}, \ldots, u_{7} \mid d_{1}, \ldots, d_{7}\right) \\
& \propto p\left(d_{1}, \ldots, d_{7} \mid u_{1}, \ldots, u_{7}\right) p\left(u_{1}, \ldots, u_{7}\right) \\
&= p\left(d_{1} \mid u_{1}, \ldots, u_{7}\right) p\left(d_{2} \mid u_{1}, u_{2}, u_{3}, u_{7}\right) \\
& \times p\left(d_{3} \mid u_{1}, u_{2}, u_{3}, u_{4}\right) p\left(d_{4} \mid u_{1}, u_{3}, u_{4}, u_{5}\right) \\
& \times p\left(d_{5} \mid u_{1}, u_{4}, u_{5}, u_{6}\right) p\left(d_{6} \mid u_{1}, u_{5}, u_{6}, u_{7}\right) \\
& \times p\left(d_{7} \mid u_{1}, u_{2}, u_{6}, u_{7}\right) p\left(u_{1}\right) p\left(u_{2}\right) \cdots p\left(u_{7}\right) .
\end{aligned}
$$

The corresponding factor graph is shown in Fig. 4, where $f_{d_{1}}$ is the factor node that corresponds to the local function $p\left(d_{1} \mid u_{1}, \ldots, u_{7}\right), \quad f_{d_{2}}$ corresponds to

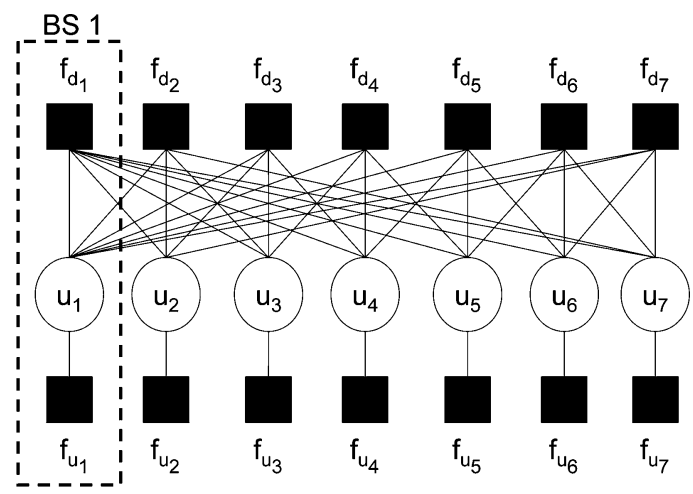

Fig. 4. Factor graph corresponding to hexagonal cellular array of seven cells.

$p\left(d_{2} \mid u_{1}, u_{2}, u_{3}, u_{7}\right)$ and so on, while $f_{u_{1}}, \ldots, f_{u_{7}}$ correspond to $p\left(u_{1}\right), p\left(u_{2}\right), \ldots, p\left(u_{N}\right)$, respectively. Note that the localized nature of the interference is reflected in the form of the factorization.

The marginal functions of interest are the conditional pdfs $p\left(u_{n} \mid \boldsymbol{d}\right), n=1, \ldots, N$, which are also Gaussian. With Gaussian $p\left(u_{n} \mid \boldsymbol{d}\right)$, the transmitted symbol from BS $n, x_{n}$, is simply the mean of $p\left(u_{n} \mid \boldsymbol{d}\right)$ and can be calculated in a distributed manner using the sum-product algorithm, which is described in the next section.

\section{B. Distributed Beamforming Using the Sum-Product Algorithm}

The sum-product algorithm is designed to compute the marginalized functions or, in the present case, the a posteriori distributions $p\left(u_{n} \mid \boldsymbol{d}\right), n=1, \ldots, N$, via message passing between the nodes in the factor graph. A comprehensive tutorial on the sum-product algorithm can be found in [45]. In this section, our aim is to show how the sum-product algorithm, operating on a factor graph that models the hexagonal cellular array, can be used as a distributed transmit beamforming algorithm.

When the sum-product algorithm is applied in a statistical inference problem, the messages passed between nodes are pdfs. For a factor graph with Gaussian variables, the messages are Gaussian pdfs, and thus they can be completely characterized by their means and variances. Furthermore, because our factor graph has loops, the sum-product algorithm is iterative in nature and does not terminate in a finite number of steps. Dropping the index $n$ for ease of notation, we denote the mean and the variance of the message passed from variable node $u$ to factor node $f$ at the $i$ th iteration, by $M_{u \rightarrow f}^{(i)}$ and $V_{u \rightarrow f}^{(i)}$, respectively. Similarly, we denote the mean and the variance of the message passed from factor node $f$ to variable node $u$ at the $i$ th iteration, by $M_{f \rightarrow u}^{(i)}$ and $V_{f \rightarrow u}^{(i)}$, respectively. We define $A(u)$ as the set of neighbors of node $u$, including node $f_{u}$, and we define $A(u) \backslash f$ to be the set of neighbors of node $u$ excluding the node $f$. Note that the neighbors of a variable node must be factor nodes and vice versa.

Considering the flooding schedule [45] where for each time instance, messages on all edges are updated simultaneously, the sum-product update equations for Gaussian factor graphs are given by [46] the following. 
Factor node to variable node

$$
\begin{aligned}
M_{f_{d} \rightarrow u}^{(i)} & =\frac{1}{h\left(f_{d}, u\right)}\left(d-\sum_{u^{\prime} \in A\left(f_{d}\right) \backslash u} h\left(f_{d}, u^{\prime}\right) M_{u^{\prime} \rightarrow f_{d}}^{(i-1)}\right) \\
V_{f_{d} \rightarrow u}^{(i)} & =\frac{1}{h^{2}\left(f_{d}, u\right)}\left(\beta+\sum_{u^{\prime} \in A\left(f_{d}\right) \backslash u} h^{2}\left(f_{d}, u^{\prime}\right) V_{u^{\prime} \rightarrow f_{d}}^{(i-1)}\right)
\end{aligned}
$$

for $i=1,2,3, \ldots$

Variable node to factor node

$$
\begin{gathered}
M_{u \rightarrow f_{d}}^{(i)}=V_{u \rightarrow f_{d}}^{(i)}\left(\sum_{f \in A(u) \backslash f_{d}} \frac{M_{f \rightarrow u}^{(i)}}{V_{f \rightarrow u}^{(i)}}\right), \quad i=1,2,3, \ldots \\
V_{u \rightarrow f_{d}}^{(i)}=\left(\sum_{f \in A(u) \backslash f_{d}} \frac{1}{V_{f \rightarrow u}^{(i)}}\right)^{-1}, \quad i=1,2,3, \ldots
\end{gathered}
$$

with initial conditions

$$
M_{u \rightarrow f_{d}}^{(0)}=0, \quad V_{u \rightarrow f_{d}}^{(0)}=1
$$

where $h\left(f_{d}, u\right)$ represents the channel gain from BS corresponding to node $u$ to the mobile in the cell corresponding to node $f_{d}$. Note that there is no need to construct the messages $M_{u \rightarrow f_{u}}^{(i)}$ and $V_{u \rightarrow f_{u}}^{(i)}$ as the factor nodes $f_{u}$ are not connected to any other nodes. Also, the $f_{u}$ nodes can be considered to be transmitting $M_{f_{u} \rightarrow u}^{(i)}=0$ and $V_{f_{u} \rightarrow u}^{(i)}=1$ by default, so there is no need to send these messages. After $i$ iterations, one obtains estimates of $\mathbb{E}[u \mid \boldsymbol{d}]$ and $\operatorname{Var}(u \mid \boldsymbol{d})$, respectively

$$
\begin{aligned}
\mathrm{E}^{(i)}(u \mid \boldsymbol{d}) & =\operatorname{Var}^{(i)}(u \mid \boldsymbol{d})\left(\sum_{f \in A(u)} \frac{M_{f \rightarrow u}^{(i)}}{V_{f \rightarrow u}^{(i)}}\right) \\
\operatorname{Var}^{(i)}(u \mid \boldsymbol{d}) & =\left(\sum_{f \in A(u)} \frac{1}{V_{f \rightarrow u}^{(i)}}\right)^{-1}
\end{aligned}
$$

for each node $u$ in the graph. The true value of $\mathrm{E}\left(u_{n} \mid \boldsymbol{d}\right)$ is the LMMSE estimate of $u_{n}$, and the global beamformer would set $x_{n}$ to be this value; the value in (25) is an approximation to $x_{n}$ obtained after $i$ steps of the sum-product algorithm.

It is easy to see that $M_{u \rightarrow f_{d}}^{(i)}$ in (23) is simply the average weighted mean estimate of $u$ from all the factor nodes except $f_{d}$. Each term in the sum on the right-hand side (RHS) of (23) is the mean obtained from a particular factor node weighted by its variance. In general, the variance reflects the confidence of the corresponding mean, with smaller variance implying higher confidence. Thus, a smaller variance in the sum corresponds to a larger weight and the overall confidence of the mean of $u$ is given by $V_{u \rightarrow f_{d}}^{(i)}$ in (24).

Equation (21) can be seen intuitively as "correcting" the mean of $u$ given the observed $d$ and the strengths of links between the factor node and the variable nodes. The strength of the link between $f_{d}$ and $u$ is given by $h\left(f_{d}, u\right)$. Likewise, the variance is also "corrected" given the observation noise variance and the link strengths as shown in (22).
In our proposed distributed beamforming algorithm, we take advantage of the fact that the variance updates in (24) and (22) do not depend on the data vector $\boldsymbol{d}$. Further, the channel states will change much more slowly than the data symbols, allowing us to make the separation of time-scale assumption that the channel states can be assumed to be fixed on the time scale of the data symbols. Following similar techniques to those presented in [47] and [48], we show in [12] that the variance updates in (24) and (22) always converge. Let $V_{u \rightarrow f_{d}}^{*}$ and $V_{f_{d} \rightarrow u}^{*}$ denote the limiting values corresponding to (24) and (22), respectively. Then the messages passed between base stations to implement the distributed beamforming algorithm are just the means of the corresponding Gaussian distributions, and we obtain the following algorithm.

\section{Sum-product beamforming algorithm}

$$
\begin{aligned}
& M_{f_{d} \rightarrow u}^{(i)}=\frac{1}{h\left(f_{d}, u\right)}\left(d-\sum_{u^{\prime} \in A\left(f_{d}\right) \backslash u} h\left(f_{d}, u^{\prime}\right) M_{u^{\prime} \rightarrow f_{d}}^{(i-1)}\right) \\
& M_{u \rightarrow f_{d}}^{(i)}=V_{u \rightarrow f_{d}}^{*}\left(\sum_{f \in A(u) \backslash f_{d}} \frac{M_{f \rightarrow u}^{(i)}}{V_{f \rightarrow u}^{*}}\right)
\end{aligned}
$$

for $i=1,2,3, \ldots$ with initial condition $M_{u \rightarrow f_{d}}^{(0)}=0$. If the algorithm is terminated after $i$ steps, then we set

$$
x_{n}^{(i)}=V_{u \rightarrow f_{d}}^{*}\left(\sum_{f \in A(u)} \frac{M_{f \rightarrow u}^{(i)}}{V_{f \rightarrow u}^{*}}\right)
$$

followed by an appropriate scaling to meet the power constraint.

\section{Convergence of the Sum-Product Beamforming Algorithm}

The sum-product algorithm is not guaranteed to converge in general, nor does it always produce the exact marginalized conditional pdf of the variable when it does converge, unless the graph is loop-free [45], and it is not loop-free in the hexagonal model.

Sufficient conditions for convergence of belief propagation for Gaussian graphical models are presented in [47]-[50]. Following similar techniques to those presented in [47] and [48], we show in [12] that the variance updates in (24) and (22) always converge, and we provide a necessary and sufficient condition for convergence of (27) and (28). The convergence condition is that the spectral radius of a certain iteration matrix is less than one, a matrix that depends on various system parameters including the path gains in the cellular network. Detailed convergence results and methods for improving convergence are presented in [51].

It is known that if the variables are Gaussian distributed, as in our case, and if convergence occurs, then the limiting value $x_{n}=\lim _{i \rightarrow \infty} x_{n}^{(i)}$ is indeed the true conditional mean, i.e., $x_{n}=\mathrm{E}\left(u_{n} \mid \boldsymbol{d}\right)$, even though $V_{u \rightarrow f_{d}}^{*}$ may not be the correct conditional variance of $u_{n}$ given $\boldsymbol{d}$ [47], [49]. Correctness of $x_{n}$ is sufficient for our problem because it is in fact the signal that should be transmitted. The limiting conditional variance of $u_{n}$ is not actually used by the beamformer, so its incorrectness is not a practical issue. 


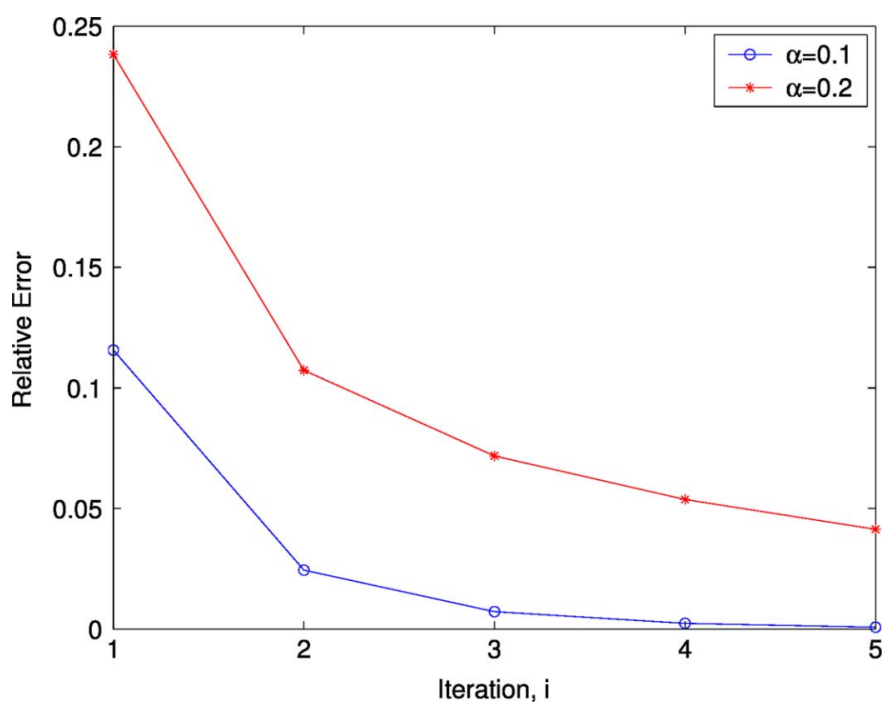

Fig. 5. Convergence of the relative error between sum-product beamformer and global beamformer.

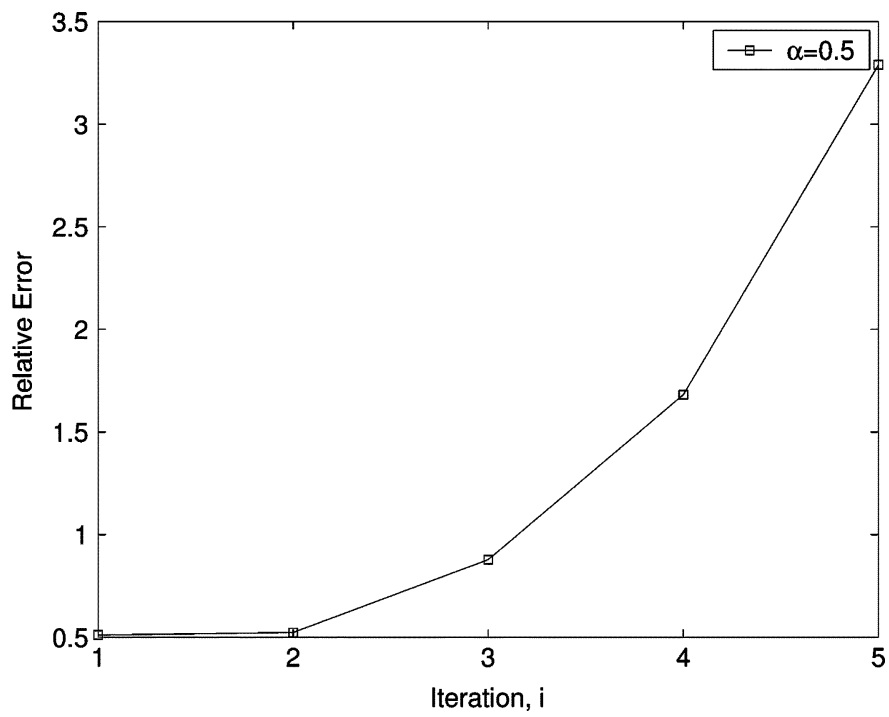

Fig. 6. Example where the sum-product beamformer fails.

Fig. 5 illustrates the convergence of the sum-product beamformer for a hexagonal network with 19 cells, same-cell gain of 1 , adjacent cell gain of $\alpha$, noise power of 1 , total global transmit power of 19 , and $\beta=1$. The plot is for a particular realization of data symbols, and the relative error plotted refers to $\frac{\left\|\boldsymbol{x}^{(i)}-\boldsymbol{x}\right\|}{\|\boldsymbol{x}\|}$, where $\boldsymbol{x}^{(i)}$ is the vector of transmitted symbols after $i$ iterations and $\boldsymbol{x}$ is the vector of transmitted symbols from the global beamformer. The convergence is geometric at a rate that is independent of the data symbols (being only dependent on the iteration matrix [51]), with $\alpha=0.1$ exhibiting faster convergence than $\alpha=0.2$, as illustrated in the figure.

Fig. 6 illustrates an example where the condition for convergence is not met. Note that it is trivial to provide a globally convergent distributed beamforming algorithm if we are willing to sacrifice optimality. The future challenge is to improve convergence without sacrificing optimality, or at least sacrificing as little as possible.

\section{CONCLUSION}

Multicell processing with cooperative BSs has tremendous potential to meet the ever increasing demand for higher data rates in cellular networks. In the downlink scenario, we show how that gain can be realized by utilizing multiple BSs in a distributed version of downlink beamforming in which information is shared only between adjacent BSs.

We first considered a simple linear array model of a cellular network, in which base stations are arranged along a line. Using the Markov structure of this model, we formulated a state-space model for the virtual LMMSE estimation problem. We adapted the Kalman smoothing framework to obtain a forward-backward, distributed beamforming algorithm. The forward-backward algorithm produces the optimal transmitted signals for each BS. However, the algorithm suffers from a delay that grows linearly with the network size.

Recognizing that only the data symbols in the local neighborhood have significant impact on the signal transmitted from a particular BS in the optimal beamformer, we then proposed a limited extent distributed beamforming algorithm. This approach allows all the base stations to operate in parallel, rather than in series. Results in [6] show that this algorithm realizes a near-optimal beamformer after a typically small number of local message passingsteps. The number of message passings needed does not grow with the network size in practice.

We also presented a distributed downlink beamforming algorithm for a 2-D hexagonal cellular array model. In this case, we remodel the hexagonal cellular array virtual uplink estimation problem as a factor graph and apply the sum-product algorithm [45] to obtain a method of distributed beamforming that generalizes the 1-D limited-extent algorithm.

Future work will be to improve the distributed beamforming algorithm, with lack of global convergence being its current weakness. Another pressing issue from a practical point of view is to investigate how clock synchronization, required to achieve coherence at the base stations, can be obtained in a distributed manner.

\section{ACKNOWLEDGMENT}

The authors would like to thank A. Grant for stimulating initial discussions, and the editor and reviewers for helpful comments on the first version of the manuscript.

\section{REFERENCES}

[1] S. Shamai and B. M. Zaidel, "Enhancing the cellular downlink capacity via co-processing at the transmitting end," in Proc. IEEE Veh. Technol. Conf. Spring, Rhodes, Greece, May 2001, vol. 3, pp. 1745-1749.

[2] S. A. Jafar and A. J. Goldsmith, "Transmitter optimization for multiple antenna cellular systems," in Proc. IEEE Int. Symp. Inf. Theory, Lausanne, Switzerland, Jun. 2002, p. 50.

[3] H. Zhang and H. Dai, "Cochannel interference mitigation and cooperative processing in downlink multicell multiuser MIMO networks," EURASIP J. Wireless Commun. Netw., no. 2, pp. 222-235, 2004.

[4] A. Ekbal and J. M. Cioffi, "Distributed transmit beamforming in cellular networks - A convex optimization perspective," in Proc. IEEE Int. Conf. Commun., Seoul, Korea, May 2005, vol. 4, pp. 2690-2694.

[5] T. Ren and R. J. La, "Downlink beamforming algorithms with inter-cell interference in cellular networks," in Proc. INFOCOM, Mar. 2005, vol. 1, pp. 47-57.

[6] B. L. Ng, J. S. Evans, S. V. Hanly, and D. Aktas, "Transmit beamforming with cooperative base stations," in Proc. IEEE Int. Symp. Inf. Theory, Sep. 2005, pp. 1431-1435. 
[7] G. J. Foschini, M. Karakayali, and R. A. Valenzuela, "Coordinating multiple antenna cellular networks to achieve enormous spectral efficiency," Inst. Electr. Eng. Proc. Commun., vol. 153, no. 4, pp. 548-555, Aug. 2006.

[8] M. Karakayali, G. J. Foschini, and R. A. Valenzuela, "Network coordination for spectrally efficient communications in cellular systems," IEEE Wireless Commun., vol. 13, no. 4, pp. 56-61, Aug. 2006.

[9] P. Marsch and G. Fettweis, "A framework for optimizing the downlink performance of distributed antenna systems under a constrained backhaul," in Proc. Eur. Wireless Conf., Paris, France, Apr. 2007 [Online]. Available: http://www.ew2007.org/papers/1569014748.pdf

[10] O. Somekh, B. Zaidel, and S. Shamai, "Sum rate characterization of joint multiple cell-site processing," IEEE Trans. Inf. Theory, vol. 53, no. 12, pp. 4473-4497, Dec. 2007.

[11] S. Jing, D. Tse, J. Hou, J. Soriaga, J. Smee, and R. Padovani, "Downlink macro-diversity in cellular networks," in Proc. IEEE Int. Symp. Inf. Theory, Chicago, IL, Jun. 2007, pp. 24-29.

[12] B. L. Ng, J. S. Evans, and S. V. Hanly, "Distributed downlink beamforming in cellular networks," in Proc. IEEE Int. Symp. Inf. Theory, Jun. 2007, pp. 6-10.

[13] Y. Liang, A. J. Goldsmith, G. Foschini, R. Valenzuela, and D. Chizhik, "Evolution of base stations in cellular networks: Denser deployment versus coordination," in Proc. IEEE Int. Conf. Commun., Beijing, China, 2008, pp. 4128-4132.

[14] S. V. Hanly and P. A. Whiting, "Information-theoretic capacity of multi-receiver networks," Telecommun. Syst., vol. 1, no. 1, pp. 1-42, Mar. 1993.

[15] A. D. Wyner, "Shannon-theoretic approach to a Gaussian cellular multiple-access channel," IEEE Trans. Inf. Theory, vol. 40, no. 6, pp. 1713-1727, Nov. 1994.

[16] L. R. Welburn, J. K. Cavers, and K. W. Sowerby, "Multiuser macrodiversity detection in asynchronous DS-CDMA systems," IEEE Trans. Wireless Commun., vol. 3, no. 2, pp. 544-554, Mar. 2004.

[17] S. V. Hanly, "Capacity and power control in spread spectrum macrodiversity radio networks," IEEE Trans. Commun., vol. 44, no. 2, pp. 247-256, Feb. 1996.

[18] A. Vanelli-Coralli, R. Padovani, J. Hou, and J. E. Smee, "Capacity of cell clusters with coordinated processing," in Proc. Inf. Theory Appl. Workshop, La Jolla, CA, Feb. 2006.

[19] N. Bacha, J. S. Evans, and S. V. Hanly, "On the capacity of MIMO cellular networks with macrodiversity," in Proc. Australian Commun. Theory Workshop, Feb. 2006, pp. 105-109.

[20] O. Somekh, B. Zaidel, and S. Shamai, "Spectral efficiency of joint multiple cell-site processors for randomly spread DS-CDMA systems," IEEE Trans. Inf. Theory, vol. 53, no. 7, pp. 2625-2637, Jul. 2007.

[21] A. Sanderovich, O. Somekh, and S. Shamai, "Uplink macrodiversity with limited backhaul capacity," in Proc. IEEE Int. Symp. Inf. Theory, Nice, France, Jun. 2007, pp. 11-15.

[22] P. Marsch and G. Fettweis, "A framework for optimizing the uplink performance of distributed antenna systems under a constrained backhaul," in Proc. IEEE Int. Conf. Commun., Jun. 2007, pp. 975-979.

[23] E. Aktas, J. S. Evans, and S. V. Hanly, "Distributed decoding in a cellular multiple-access channel," in Proc. IEEE Int. Symp. Inf. Theory, Chicago, IL, Jun. 2004, p. 484.

[24] O. Shental, A. Weiss, N. Shental, and Y. Weiss, "Generalized belief propagation receiver for near optimal detection of two dimensional channels with memory," in Proc. IEEE Inf. Theory Workshop, Oct. 2004, pp. 225-229.

[25] E. Aktas, J. S. Evans, and S. V. Hanly, "Distributed decoding in a cellular multiple access channel," IEEE Trans. Wireless Commun., pp. 241-250, Jan. 2008

[26] C. B. Peel, B. M. Hochwald, and A. L. Swindlehurst, "A vector-perturbation technique for near-capacity multi-antenna multi-user communication-Part I: Channel inversion and regularization," IEEE Trans. Commun., vol. 53, no. 1, pp. 195-202, Jan. 2005.

[27] S. Thoen, L. V. der Perre, and M. Engels, "Improved adaptive downlink for OFDM/SDMA-based wireless networks," in Proc. Veh. Technol. Conf. Spring, May 2001, vol. 1, pp. 707-711.

[28] T. Haustein, M. Schubert, and H. Boche, "On power reduction strategies for the multi-user downlink with decentralized receivers," in Proc. Veh. Technol. Conf. Spring, Apr. 2003, vol. 2, pp. 1001-1011.
[29] G. Caire and S. Shamai, "On the achievable throughput of a multiantenna Gaussian broadcast channel," IEEE Trans. Inf. Theory, vol. 49 , no. 7, pp. 1691-1706, Jul. 2003.

[30] M. Joham, K. Kusume, M. H. Gzara, W. Utschick, and J. A. Nossek, "Transmit Wiener filter for the downlink of TDDDS-CDMA systems," in Proc. IEEE 7th Int. Symp. Spread-Spectrum Tech. Appl., Prague, Czech Republic, Sep. 2002, vol. 1, pp. 9-13.

[31] L.-U. Choi and R. D. Murch, "Transmit-preprocessing techniques with simplified receivers for the downlink of MISO TDD-CDMA systems," IEEE Trans. Veh. Technol., vol. 53, no. 2, pp. 285-295, Mar. 2004.

[32] A. N. Barreto and G. Fettweis, "Capacity increase in the downlink of spread spectrum systems through joint signal precoding," in Proc. Int. Conf. Commun., Helsinki, Finland, Jun. 2001, vol. 4, pp. 1142-1146.

[33] F. Kowalewski and P. Mangold, "Joint predistortion and transmit diversity," in Proc. IEEE Global Telecommun. Conf., Nov. 2000, vol. 1, pp. $245-249$.

[34] B. R. Vojčić and W. M. Jang, "Transmitter preceding in synchronous multiuser communications," IEEE Trans. Commun., vol. 46, no. 10, pp. 1346-1355, Oct. 1998.

[35] A. Wiesel, Y. C. Eldar, and S. Shamai, "Linear precoding via conic optimization for fixed MIMO receivers," IEEE Trans. Signal Process. vol. 54, no. 1, pp. 161-176, Jan. 2006.

[36] M. Schubert and H. Boche, "Solution of the multiuser downlink beamforming problem with individual SINK constraints," IEEE Trans. Veh. Technol., vol. 53, no. 1, pp. 18-28, Jan. 2004.

[37] H. Boche and M. Schubert, "A general duality theory for uplink and downlink beamforming," in Proc. IEEE Veh. Technol. Conf. Fall, Sep. 2002, pp. 87-91.

[38] B. L. Ng, J. S. Evans, and S. V. Hanly, "Distributed linear multiuser detection in cellular networks based on Kalman smoothing," in Proc. IEEE Global Telecommun. Conf., Dallas, TX, Dec. 2004, vol. 1, pp. 134-138.

[39] B. L. Ng, J. S. Evans, S. V. Hanly, and A. J. Grant, "Information capacity of Wyner's cellular network with LMMSE receivers," in Proc. IEEE Int. Conf. Commun., Paris, France, Jun. 2004, vol. 1, pp. 583-587.

[40] D. N. C. Tse and P. Viswanath, "Downlink-uplink duality and effective bandwidths," in Proc. IEEE Int. Symp. Inf. Theory, Lausanne, Switzerland, Jun. 2002, p. 52.

[41] E. Visotsky and U. Madhow, "Optimum beamforming using transmit antenna arrays," in Proc. IEEE 49th Veh. Technol. Conf., May 1999, vol. 1, pp. 851-856.

[42] F. Rashid-Farrokhi, K. J. R. Liu, and L. Tassiulas, "Transmit beamforming and power control for cellular wireless systems," IEEE J. Sel. Areas Commun., vol. 16, no. 8, pp. 1437-1450, Oct. 1998.

[43] S. M. Kay, Fundamentals of Statistical Signal Processing-Estimation Theory. Englewood Clifs, NJ: Prentice-Hall, 1993.

[44] D. C. Fraser and J. E. Potter, "The optimum linear smoother as a combination of two optimum linear filters," IEEE Trans. Autom. Control, vol. 14, no. 4, pp. 387-390, Aug. 1969.

[45] F. R. Kschischang, B. J. Frey, and H.-A. Loeliger, "Factor graphs and the sum-product algorithm," IEEE Trans. Inf. Theory, vol. 47, no. 2, pp. 498-519, Feb. 2001.

[46] B. J. Frey, , S. A. Solla, T. K. Leen, and K. R. Müller, Eds., "Local probability propagation for factor analysis," in Advanced in Neural Information Processing Systems 12. Cambridge, MA: MIT Press, 2000, pp. $442-448$.

[47] P. Rusmevichientong and B. V. Roy, "An analysis of belief propagation on the turbo decoding graph with Gaussian densities," IEEE Trans. Inf. Theory, vol. 47, no. 2, pp. 745-765, Feb. 2001.

[48] E. C. Liu and J. M. F. Moura, "Fusion in sensor networks: Convergence study," in Proc. IEEE Int. Conf. Acoust. Speech Signal Process., Montreal, QC, Canada, May 2004, vol. 3, pp. 865-868.

[49] Y. Weiss and W. T. Freeman, "Correctness of belief propagation in Gaussian graphical models of arbitrary topology," Neural Comput., vol. 13, pp. 2173-2200, 2001.

[50] D. M. Malioutov, J. K. Johnson, and A. S. Willsky, "Walk-sums and belief propagation in Gaussian graphical models," J. Mach. Learn. Res., vol. 7, pp. 2031-2064, 2006.

[51] B. L. Ng, "Cellular networks with cooperating base stations: Performance analysis and distributed algorithms," Ph.D. dissertation, Dept. Electr. Electron. Eng., Univ. Melbourne, Melbourne, Australia, 2007. 